\title{
Searching for helium in the exosphere of HD 209458b^
}

\author{
C. Moutou ${ }^{1}$, A. Coustenis ${ }^{2}$, J. Schneider ${ }^{3}$, D. Queloz ${ }^{4}$, and M. Mayor ${ }^{4}$ \\ 1 Laboratoire d'Astrophysique de Marseille, BP 8, 13376 Marseille Cedex 12, France \\ 2 LESIA, Observatoire de Meudon, Pl. J. Janssen, 92195 Meudon Cedex, France \\ 3 LUTH, Observatoire de Meudon, Pl. J. Janssen, 92195 Meudon Cedex, France \\ ${ }^{4}$ Observatoire de Genève, 51 ch. des Maillettes, 1290 Sauverny, Switzerland
}

Received 16 December 2002 / Accepted 16 April 2003

\begin{abstract}
Atmospheric models of the extrasolar, close-in giant planet HD 209458b predict strong absorption features from alkali metals (Seager \& Sasselov 2000; Brown 2001). This was confirmed by the discovery of NaI by HST observations (Charbonneau et al. 2002). In this study we focus on the search for the helium absorption feature at $10830 \AA$, also predicted to be among the strongest ones. Helium is a major component of the planet's exosphere, for which models are yet not as robust as atmosphere models. One full transit was observed with the VLT/ISAAC instrument. We do not report the detection of the HeI feature. The data set is strongly affected by instrumental fringing, at a level up to 5\% in the extracted spectra. After filtering, a residual noise of the order of $0.2 \%$ remains. An upper limit of the HeI line was derived, which further constrains future models of the HD 209458b planet exosphere. This upper limit, in terms of the feature depth, is $0.5 \%$ at $3 \sigma$ for a $3 \AA$ bandwidth. Prospects are proposed to lower the detectability limit; the ultimate detectability limit with ISAAC in the absence of electronic fringing and in ideal atmospheric conditions could be as low as a line depth of $0.1 \%$ ( $3 \AA$ width, $3 \sigma$ ).
\end{abstract}

Key words. stars: planetary systems - infrared: stars - planets and satellites: general

\section{Introduction}

The knowledge on extra-solar planets is rapidly improving with the progress of radial velocity surveys (Marcy et al. 2002; Udry et al. 2002 and references therein). So far, a single planetary system among the $\sim 100$ discovered has the projected geometry favourable to the observation of the planetary transit. The shallow dip produced on the light curve of the star HD 209458 (Charbonneau et al. 2000) by the planet allowed a direct determination of the planet radius and confirmed for the first time the planetary nature of the companion, a gas-giant planet probably rich in hydrogen and helium (Mazeh et al. 2000; Burrows et al. 2000; Cody \& Sasselov 2002). This system has further been the ideal target for many follow-up observations, aiming at very high photometric accuracy (Brown et al. 2001a) or high signal-to-noise ratio, high-resolution optical spectra for the search of exoplanetary atmospheric signatures (Bundy \& Marcy 2000; Rauer et al. 2000; Moutou et al. 2001; Brown et al. 2002; Richardson et al. 2003). Charbonneau et al. (2002) detected the NaI doublet at $589.3 \mathrm{~nm}$ with the STIS instrument onboard HST. With a spectrophotometric accuracy of a few $10^{-4}$ in a narrow spectral bin centred on the NaI doublet, the planetary transit appeared deeper relative to adjacent bins,

Send offprint requests to: C. Moutou, e-mail: Claire.Moutou@oamp.fr

* Based on data acquired on the Very Large Telescope at Paranal Observatory, ESO Chile. free of planetary signatures, with a significance of $4.1 \sigma$. No confirmation of this result has been made so far; the possibility to get similar accuracy from a ground-based observatory is strongly limited in this spectral range due to telluric contamination; the detection of planetary $\mathrm{NaI}$ in transmission would need a careful monitoring of telluric features during the transit sequence.

The system OGLE-TR-56 could harbour another transiting planet, with an extremely short period, following the recent interpretation by Konacki et al. (2003), but any follow-up observations will be difficult due to the star brightness.

Atmospheric models provided a prediction on the transmission spectrum of the system (Seager \& Sasselov 2000; Brown 2001b; Hubbard et al. 2001). The cloud top, the atmospheric metallicity and the wind profiles are among the important parameters which are poorly constrained by our present knowledge and for which no observational data is available yet. Such models do not yet take into account the complex photochemistry, interactions of the exosphere with the stellar wind, atmospheric circulation and photoionization taking place in the highest levels of the atmosphere. Finally, the authors find that the strongest optical features arise from alkali metals, NaI, KI and HeI. In the near-IR, the transmission spectrum is dominated by molecular features, mainly $\mathrm{H}_{2} \mathrm{O}, \mathrm{CO}$, $\mathrm{CH}_{4}$ (Brown 2001b). The abundance of $\mathrm{NaI}$ in the atmosphere of HD 209458b derived from HST observations (Charbonneau et al. 2002) turns to be significantly smaller (by a factor of 3 ) 
than the abundance predicted by these first models. New effects are thus progressively inserted into the atmospheric models, such as the photoionization of NaI, non-LTE processes, and the presence of high-altitude clouds (Barman et al. 2002; Fortney et al. 2002). The resulting abundance of NaI is better reproduced by the second generation models, which however still lack additionnal observational constraints.

The HeI feature at $10830 \AA$ is predicted to be one of the deepest absorption lines of the diffuse and extended exosphere in the optical and near-IR transmission spectrum of HD 209458 (Seager \& Sasselov 2000). Recently, Vidal-Madjar et al. (2003) announced the detection of a $15 \%$ deep absorption in the Ly $\alpha$ line, during a transit of HD 209458b, which is interpreted in terms of evaporated atmosphere. The goal of the present study is to search for this feature in the spectrum of HD 209458, during a transit of the planet.

\section{Observations}

The system HD 209458 was observed using the spectroscopic mode of ISAAC on the VLT. The observed planet transit occurred on the 15th of June 2001, with centre at 08:31 UT. The observing sequence starts $35 \mathrm{~min}$ before the first contact, then covers the whole transit duration ( 3 hours) and ends 45 min after the last contact. Two nights later, the star was observed again for comparison during the same slot of time, starting $20 \mathrm{~min}$ earlier. Due to the northern declination of HD 209458, it is not possible to follow this object long enough from the VLT to get off-transit spectra before or after a transit. This is the reason why the off-transit spectrum series were acquired two nights later. It also follows a similar airmass sequence as the on-transit observation series, and do not carry the risk of having planetary material in the line of sight, should the exosphere be widely extended. Two neighbour stars were also observed during both nights, prior to the observation of HD 209458: HD 209008 (B3III) and HD 208669 (G0). They allow the identification of telluric and stellar contributions. HD 209458 being of spectral type G2V, the spectrum of HD 208669 is relatively similar, while the spectrum of HD 209008 is comparatively free of most stellar features and mainly exhibits telluric features. Unfortunately it was not possible to get the spectra of both comparison stars during and after the transit sequence. Consequently, the spectra of comparison stars could not be used for the removal of telluric features, but rather for the simple identification of these features. Table 1 summarizes the observing material.

Due to the efficiency of the VLT/ISAAC instrument and the brightness of the main target $(V=7.65)$, short exposure times were used and many frames of HD 209458 could be recorded: 158 exposures of $80 \mathrm{~s}$ during the transit night, and the same amount during the off-transit night. Individual detector integration time is $20 \mathrm{~s}$, which means that 4 frames were co-added 158 times by the detector controller; this allows us to reduce the noise without reaching saturation. We performed mediumresolution spectroscopy with a $0.6^{\prime \prime}$ slit, resulting in a resolving power of 5800. The spectral range covered (10 600-11000 is centred close to the expected HeI feature. Nodding and
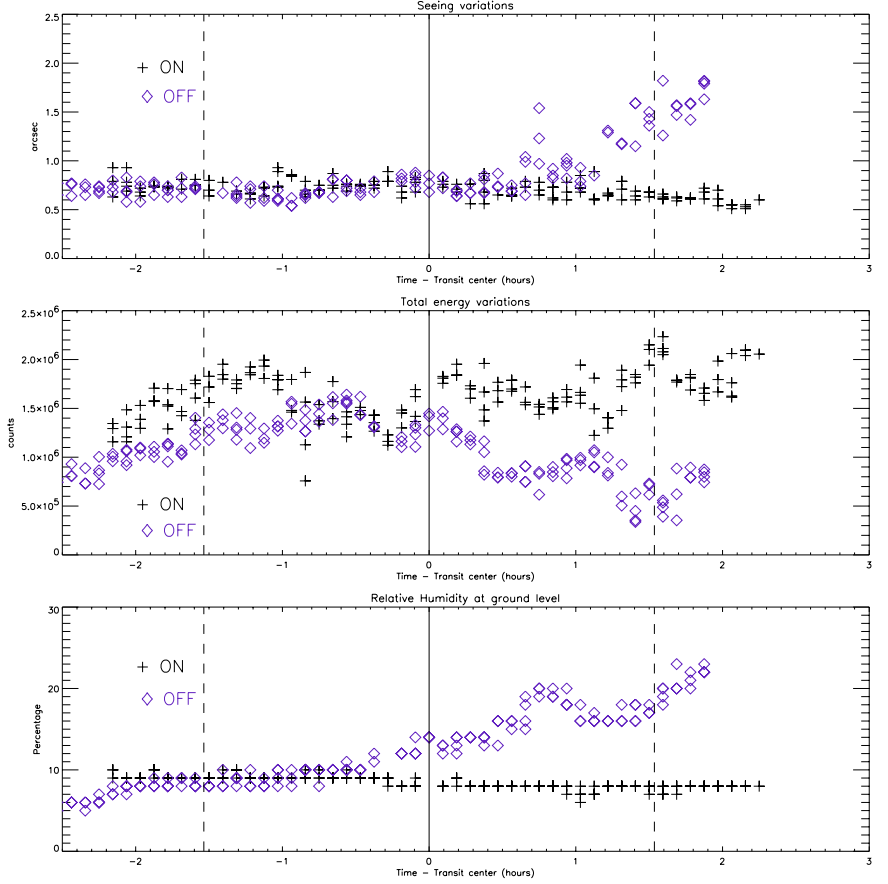

Fig. 1. Variations with time of the atmospheric seeing (top), of the total energy contained in the spectrum of HD 209458 (middle) and of the relative ambient humidity at low layers during the observing sequence (bottom). Diamonds (plus signs) show the behavior for the off-transit (respectively on-transit) night. Data quality is good except during the last $45 \mathrm{~min}$ of the off-transit night. The $X$ axis is in relative time where the origin is the transit centre time. The vertical continuous lines show the transit centre time while both dashed lines show the first and last contact of the HD 209458 eclipse as it occurred on June 15th, 2001.

jittering along the slit allowed for an efficient cancellation of the nightsky background emission.

Flatfield frames and lamp exposures were acquired during daytime and used for the correction of the science frames and for the spectral calibration. However, several weeks after the observations, Paranal Observatory noticed that an odd-even column effect occured in a time slot containing our data set, and performed new calibration files. The flatfield used for data reduction comes from the latter set of calibration files, taken several weeks after the observations. The internal calibration frames are thus far from optimal and may cause additional noise.

The transit night was recorded as photometric, while parts of the off-transit night were of slightly poorer transparency. Figure 1 (top) shows the variations of the atmospheric seeing which occured during both observing sequences. The total energy contained in the spectrum, well-correlated with the seeing, proves to be well representative of the data quality (middle plot of Fig. 1). The spectral energy is also linked to the airmass during observations, which was minimal close to transit centre (Table 1). Also the ambient humidity at the telescope level was recorded and its variations are also plotted against time, as this parameter may be of importance for IR observations (bottom of Fig. 1). Finally, it appears that both nights were of similar quality during most of the observing sequence. Only the last $45 \mathrm{~min}$ were up to $40 \%$ worse during the off-transit night. This analysis of data quality was done in order to select the best frames, 


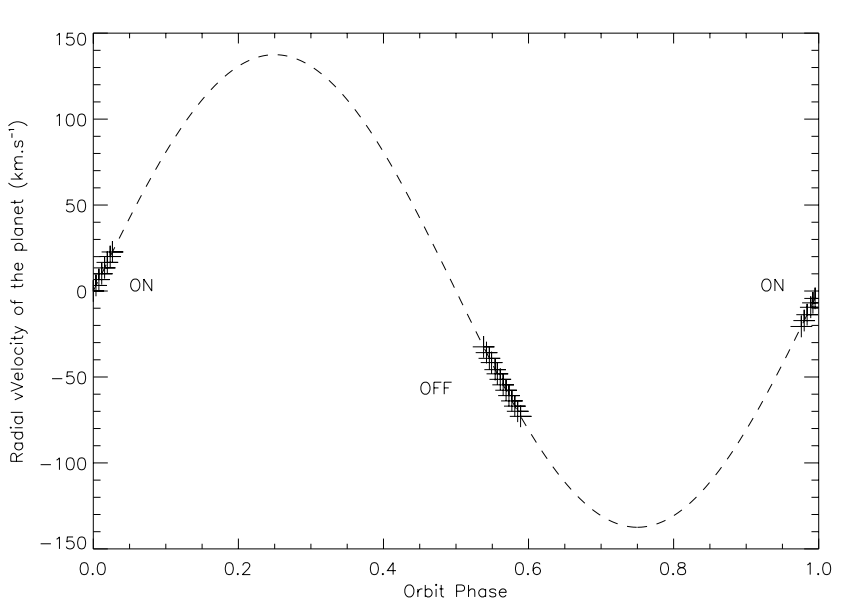

Fig. 2. The theoretical radial velocity curve of the planet is shown. Superimposed (cross symbols) are the orbital phases when a spectrum was acquired with VLT/ISAAC. The corresponding $R V$ values were used to recenter the spectra in the velocity frame of the planet.

with the condition that it doesn't disrupt the time sampling. At the end, only a few frames were rejected before combination, as a consequence of the rather good conditions during both nights. The criterion used for the data selection was the total spectral energy.

The transit sequence is also illustrated in Fig. 1, where the continuous line shows the transit centre and the two dashed lines point to the times of first and last contact (following the ephemeris in Brown et al. 2001a). During the transit observing sequence, the radial velocity of the planet has changed from -16 to $+18 \mathrm{~km} \mathrm{~s}^{-1}$ (Fig. 2), which corresponds to a motion of $1.24 \AA$ of the spectral line, in total. In the wavelength frame of the star at transit centre, the feature is expected at the position of $10830.3 \AA$.

\section{Data reduction}

Spectra were first combined into 14 subsets, following the time sequence, in order to increase the signal-to-noise ratio while keeping part of the temporal sequence. Within each subset, the spectra are located at the same position on the detector. A similar grouping was done for the off-transit spectra in equivalent time slots; this allows in principle to cancel out part of the atmospheric signatures since the airmass sequence is similar during both on- and off-transit nights. An average spectrum of HD 209458 was also calculated in order to perform the line identification by comparison with the solar spectrum (Sect. 4).

The shift-and-add technique is then used for combining 2D frames, through the Eclipse/ISAAC pipeline recipes (Devillard 2001). Two-dimensional distortions are efficiently corrected for with the aid of a lamp spectrum and star trace images. The eclipse data reduction routine also subtracts the sky background, corrects for flatfield and extracts the spectrum on 10 pixels.

The characteristics that are most noticeable are the presence of high-frequency fringes which strongly affect the extracted spectra (Fig. 3). The pixel-to-pixel variations are of
Table 1. Summary of the observation sequences of the planetary system HD 209458. On-transit spectra have been acquired during night June 15th, 2001 while off-transit spectra have been taken on June 17th. $A M$ stands for airmass and SNR for signal-to-noise ratio. Orbital phase of the planet and its corresponding relative radial velocity is given with reference to the transit centre (phase and $R V=0$ ).

\begin{tabular}{lccccc}
\hline \hline Target & $\begin{array}{c}\text { Time of } \\
\text { obs (UT) }\end{array}$ & $\begin{array}{c}\text { Orbit } \\
\text { phase }\end{array}$ & $A M$ & $S N R$ & $\begin{array}{c}\text { Planet } R V \\
\mathrm{~km} \mathrm{~s}^{-1}\end{array}$ \\
\hline ON 1 & $06: 30$ & 0.976 & 1.90 & 652 & 16.33 \\
ON 2 & $06: 50$ & 0.980 & 1.75 & 638 & 13.62 \\
ON 3 & $07: 10$ & 0.984 & 1.65 & 666 & 10.90 \\
ON 4 & $07: 30$ & 0.989 & 1.54 & 678 & 7.49 \\
ON 5 & $07: 50$ & 0.992 & 1.48 & 658 & 5.45 \\
ON 6 & $08: 10$ & 0.995 & 1.43 & 653 & 3.40 \\
ON 7 & $08: 30$ & 0.000 & 1.39 & 664 & -0.015 \\
ON 8 & $08: 50$ & 0.0037 & 1.38 & 683 & -2.54 \\
ON 9 & $09: 10$ & 0.0077 & 1.38 & 675 & -5.27 \\
ON 10 & $09: 30$ & 0.0116 & 1.38 & 673 & -7.93 \\
ON 11 & $09: 50$ & 0.0156 & 1.40 & 665 & -10.66 \\
ON 12 & $10: 10$ & 0.0193 & 1.44 & 696 & -13.17 \\
ON 13 & $10: 30$ & 0.0233 & 1.49 & 687 & -15.88 \\
ON 14 & $10: 50$ & 0.0264 & 1.53 & 684 & -17.98 \\
\hline OFF 1 & $06: 00$ & 0.538 & 2.1 & 538 & 25.71 \\
OFF 2 & $06: 20$ & 0.542 & 1.90 & 566 & 28.36 \\
OFF 3 & $06: 40$ & 0.546 & 1.75 & 591 & 30.99 \\
OFF 4 & $07: 00$ & 0.549 & 1.64 & 612 & 32.95 \\
OFF 5 & $07: 20$ & 0.554 & 1.55 & 625 & 36.19 \\
OFF 6 & $07: 40$ & 0.557 & 1.48 & 631 & 38.12 \\
OFF 7 & $08: 00$ & 0.561 & 1.44 & 607 & 40.67 \\
OFF 8 & $08: 20$ & 0.565 & 1.40 & 557 & 43.19 \\
OFF 9 & $08: 40$ & 0.569 & 1.38 & 522 & 45.69 \\
OFF 10 & $09: 00$ & 0.573 & 1.38 & 560 & 48.16 \\
OFF 11 & $09: 20$ & 0.577 & 1.38 & 539 & 50.59 \\
OFF 12 & $09: 40$ & 0.581 & 1.40 & 504 & 53.00 \\
OFF 13 & $10: 00$ & 0.585 & 1.43 & 430 & 55.37 \\
OFF 14 & $10: 20$ & 0.589 & 1.48 & 382 & 57.70 \\
\hline & & & & & \\
\hline
\end{tabular}

variable amplitude along the spectrum, being reduced towards the red. The amplitude of the defect also changes from one quadrant to the other. In the upper right (resp. left) quadrant, the amplitude is $2.5 \%(4.9 \%)$ while in the bottom right (resp. left) quadrant, it reaches $1.5 \%(2.4 \%)$. The HeI feature is expected to fall in the right quadrant, close to its left border, near the centre of the chip, at a location where the fringing is significant. This "odd-even column effect" was randomly observed on ISAAC, more frequently when using short integration times in spectroscopy (i.e., like in this case). It can, in principle, be removed by filtering out post-processing. The spectra were extracted on each quadrant individually in order to get rid more efficiently of the odd-even effect. A low-pass digital filter was optimised for each quadrant by the minimization of the residual local standard deviation. The subsequent effect of fringe removal is a slight smoothing of the spectrum. The residual noise, measured on the difference between both quadrants, is of the order of $0.2 \%$ in the $10830 \AA$ zone. This fringing 

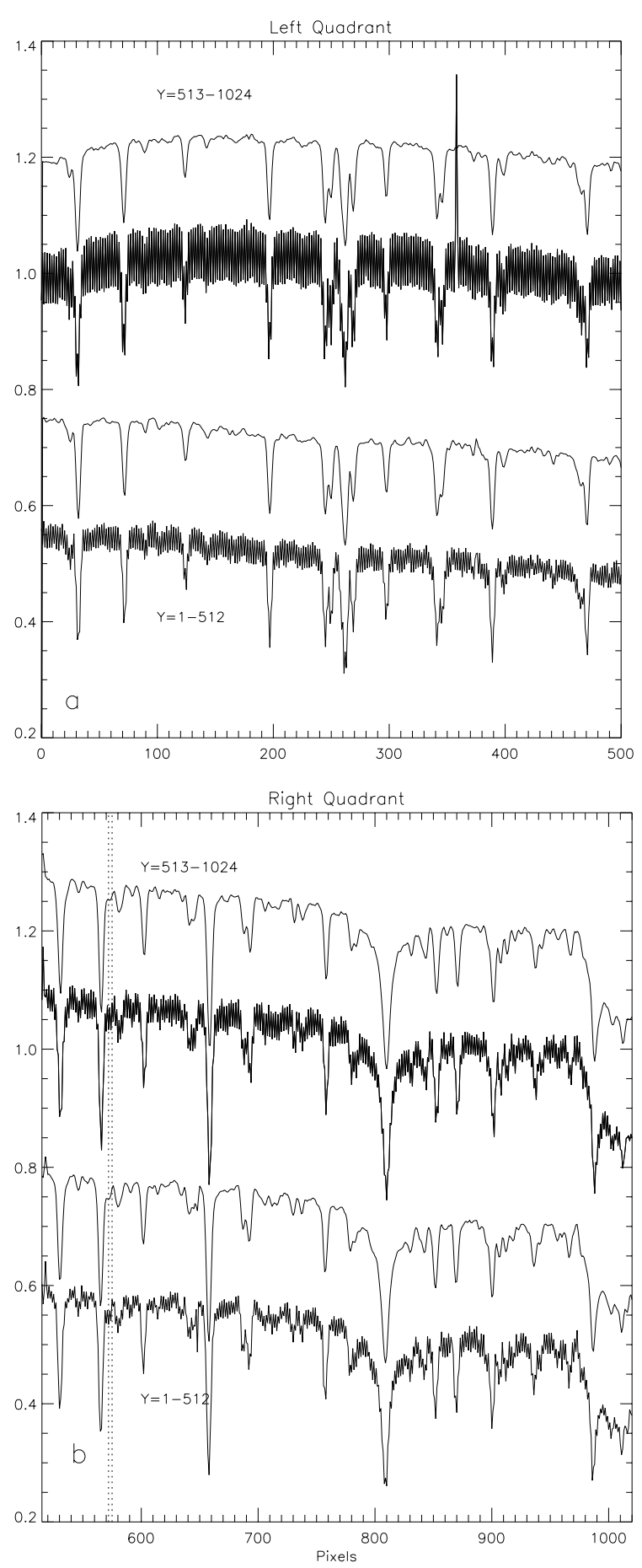

Fig. 3. The filtering of the odd-even column effect is illustrated. a) the left quadrant and b) the right quadrant of the Hawaii detector. The fringes have between 2 and 5\% amplitude. On each plot, both spectra at the top correspond to the upper part of the detector $(Y=513-1024)$ while both spectra shifted to the bottom correspond to the lower section $(Y=1-512)$. Abscissas are $X$ pixels. The spectra with reduced noise are the results of the filtering, with a 0.2 vertical offset for clarity. The position of the expected HeI feature is marked with dotted lines.

effect could be the main limitation in our search for the HeI feature. Figure 3 shows the cancellation of the fringing pattern in each detector quadrant. Out of the off-transit spectra, of slightly lower quality due to worse atmospheric conditions,

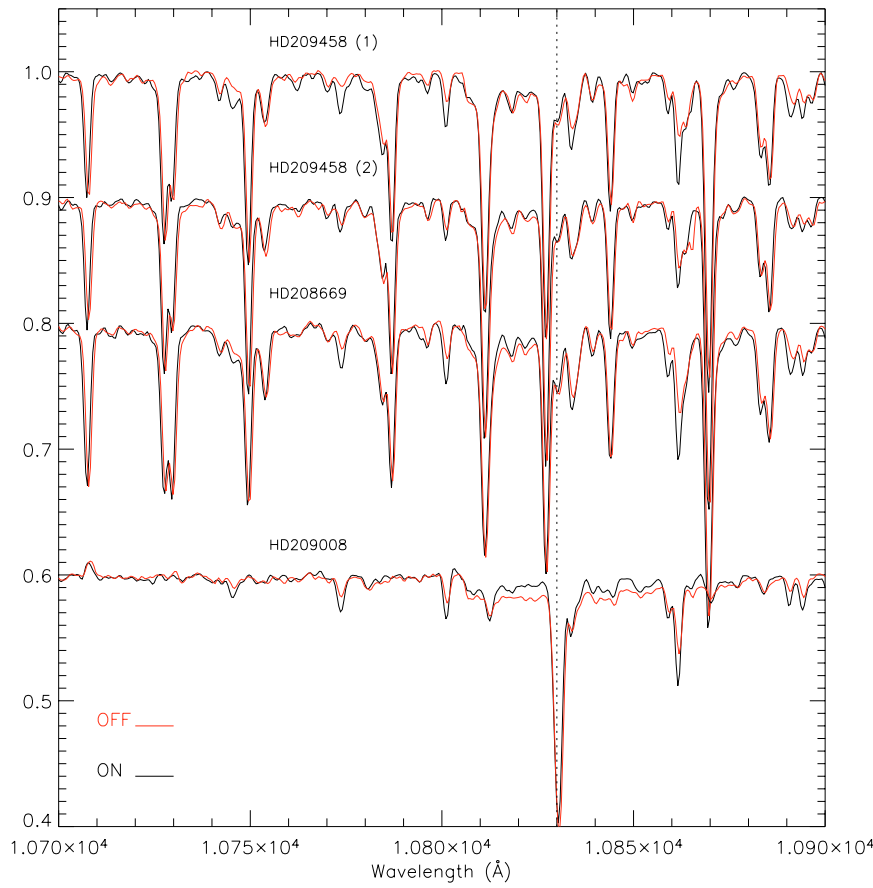

Fig. 4. Reduced and extracted spectra of HD 209458 (divided in time into the consecutive period (1) and period (2)), HD 208669 and HD 209008, from top to bottom, during both on-transit (thick line) and off-transit (thin line) nights. The plot shows the domain 10700$10900 \AA$ A. The spectra, all normalized to unity, are shifted in $Y$ for more visibility. The position of the expected HeI feature is marked with dotted lines.

two reference spectra were produced, one for the upper half of the detector, and one for the lower part. These are the reference spectra, of signal-to-noise ratio 1200 (lower quadrant, 5 spectra summed up) and 1400 (upper quadrant, 6 spectra summed up), to which the 14 on-transit spectra were compared afterwards.

The on-transit and off-transit spectra were normalized to unity. No attempt to fit the continuum was made to avoid introducing artefacts, but it seemed reasonable to directly compare on and off spectra from the same quadrant, after an identical filtering.

The resulting signal-to-noise ratio on the $2 \times 14$ subsets of spectra after fringe removal is shown in Table 1.

\section{Line identification}

The resulting spectra are shown in Fig. 4. From top to bottom are shown:

- The spectrum of HD 209458, during the first period of the on- and off-transit nights (average of subset spectra 1 to 8 ).

- The spectrum of HD 209458, during the second period of both nights (average of subset spectra 9 to 14).

- The spectrum of HD 208669, the G0 comparison star, during both nights, before the transit sequence.

- The spectrum of HD 209008, the B3 comparison star, during both nights, before the transit sequence.

It is obvious that many differences are visible between the on- and off-transit spectra at similar epochs. The observed 


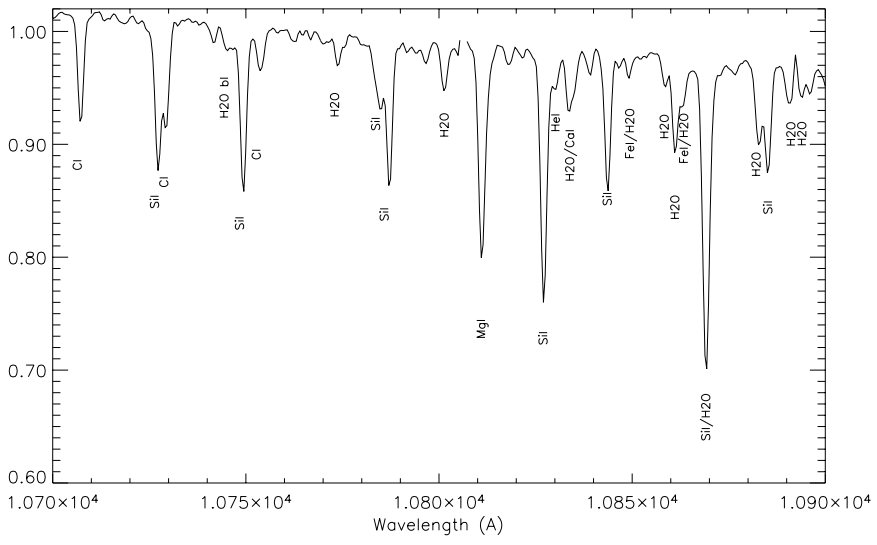

Fig. 5. Identification of stellar and telluric lines on the HD 209458 spectrum. The identification was done based on the solar spectrum (Delbouille et al. 1973). "bl" indicates a blending of several lines. This illustrates that all observed variations between spectra taken on the 15th and 17th of June, 2001 arise from $\mathrm{H}_{2} \mathrm{O}$ telluric lines.

variations are of the order of 1 to $4 \%$, much larger than the local noise. However, these variations affect the spectra of HD 209458 as well as the spectra of the comparison stars, testifying against an origin of the observed effect in the exoplanet atmosphere. Indeed, the spectral lines in the scanned region were all identified and it turns out that the varying features were all of telluric water origin. Figure 5 shows the identification of the stellar and telluric lines; this was done based on the solar spectrum, as the spectral type of HD 209458 is rather close to the Sun's. Note that the content of telluric $\mathrm{H}_{2} \mathrm{O}$ is larger during the on-transit night at a similar airmass value - as all $\mathrm{H}_{2} \mathrm{O}$ features appear deeper in on-transit spectra - a result which was not predictable from the ambient humidity recorded close to the ground (bottom of Fig. 1). Humidity of the Earth atmosphere at higher altitudes is probably seen here. The variation of water lines is of the order of $1 \%$ during the on-transit night, only $0.2 \%$ during the off-transit night, and more than $2 \%$ from one night to the other.

The possibility that the higher content of $\mathrm{H}_{2} \mathrm{O}$ in the ontransit spectra arises from the exoplanet atmosphere can be ruled out, considering that different line velocities should then be observed.

The stellar HeI feature is identified in our spectrum at $10830 \AA$ as a weak line in the wing of the deeper SiI feature. Possible blends of the stellar HeI may occur at this spectral resolution, with stellar $\mathrm{CN}, \mathrm{CaI}$ and telluric $\mathrm{H}_{2} \mathrm{O}$ lines, though $\mathrm{HeI}$ should be the main contribution at this wavelength.

\section{Upper limit on the planetary Hel feature}

\subsection{Formation of the $\mathrm{Hel}$ feature}

The HeI absorption line at $10830 \AA$ results from the transition $1 \mathrm{~s} 2 \mathrm{~s}^{3} \mathrm{~S}-1 \mathrm{~s} 2 \mathrm{p}^{3} \mathrm{P}^{0}$, both triplet states. To populate the metastable ${ }^{3} \mathrm{~S}$ state, it is necessary to invoke first the photoionisation of helium and the consecutive recombination (Andretta \& Jones 1997). The line is only a few percent deep in the Sun spectrum and is expected to contribute even less to the spectrum of HD 209458 (Seager \& Sasselov 2000). The strength of the
$10830 \AA$ HeI feature originated in the planet envelope is mostly induced by the extreme UV flux, at wavelengths smaller than $50.4 \mathrm{~nm}$, and therefore strongly linked to the star coronal activity. Should this flux be precisely estimated, then the measurement of the HeI feature would allow us to better constrain the atmospheric models of this giant planet. The intrinsic width of the HeI feature is dominated by collisional events with accelerated electrons and protons of the stellar wind; the order of magnitude of the bandwidth is estimated to be $3 \AA$ (Seager \& Sasselov 2000).

The model also predicts a relative flux of the order of $10^{3}$ in the HeI feature versus the local continuum. Diluted by a factor $3.6 \times 10^{-4}$ to account for the geometrical cross-section of the atmosphere projected on the star disk (Seager \& Sasselov 2000 ), this leads to a $0.25 \%$ deep feature. This constitutes a lower limit since the exosphere is more spatially extended than the bounded atmosphere.

Also, the HeI feature moves on the star spectrum, due to the orbital motion of the planet. From the beginning of the transit observation sequence to its end, the motion is of the order of $34 \mathrm{~km} \mathrm{~s}^{-1}$, which corresponds to $\pm 0.61 \AA$ shifts towards the blue before transit centre, and towards the red afterwards, in the star velocity frame. We have recentred the on-transit spectra in the planet velocity frame. This consequently slightly broadens the stellar lines but the possible planet line is less diluted.

\subsection{Detectability limit}

The search for a HeI feature originating in the planet exosphere has been conducted with the following methods: (1) visual inspection of the on- and off-transit spectra and of the difference of both spectra; (2) rebinning of the spectra in spectral intervals similar to the expected feature width, and comparison of the temporal behaviour at $10830 \AA$, differentially to neighbour spectral bins and to off-transit data, and its comparison with the same function out-of-transit (similarly to the method used by Charbonneau et al. 2002); (3) introduction of a fake feature of a varying amplitude and width and $3 \sigma$-detection of this feature in our data. The second method proved not optimal since the fluctuation of telluric features easily dominates any variation in our spectrum series. Also, the signal-to-noise ratio is not sufficient in individual spectra to reach the best detectability. Better results were obtained by visual inspection of a carefully built transmission spectrum, i.e., where all high-quality transit spectra are summed up and where the off-transit reference has been convolved, shifted and subtracted. These operations were optimized by $\chi^{2}$ minimization. The standard deviation of the transmission spectrum is then calculated as follows, taking into account the line width:

$\sigma \sim \sigma_{\text {cont }} * D * \sqrt{(2 * F W H M / D)}$

where $D$ is the dispersion, $\sigma_{\text {cont }}$ is the standard deviation of the spectrum continuum and $F W H M$ is the line width (Tresse et al. 1999). Finally, the third method allows the treatment of the fake feature along the reduction process and an active control of the line parameters; the detection of this fake feature is then naturally decided using the $3 \sigma$ level limit in the average spectrum of both quadrants. The equivalent width of the 
Table 2. Upper limit detectability (expressed in number of $\sigma$ ) for various combinations of width and depth for a fake HeI absorption line.

\begin{tabular}{lcccc}
\hline \hline Depth & $F W H M$ & SNR (up) & SNR (down) & SNR (avg) \\
\hline $1 \%$ & $3 \AA$ & 8.0 & 6.0 & 5.8 \\
$0.5 \%$ & $3 \AA$ & 3.8 & 2.8 & 2.6 \\
$0.5 \%$ & $7 \AA$ & 5.3 & 3.8 & 3.6 \\
$0.4 \%$ & $6 \AA$ & 3.4 & 2.5 & 2.3 \\
$0.3 \%$ & $10 \AA$ & 3.6 & 2.6 & 2.4 \\
\hline
\end{tabular}

fake feature is compared to the above-defined $\sigma$. Table 2 gives the combination of parameters for a line at $10830 \AA$ to be detected. A feature with a $0.5 \%$ central depth and $3 \AA$ broadening would be $3 \sigma$-detected. If twice broader, the detected line could be $20 \%$ shallower.

In Fig. 6, the derived on-transit spectrum is superimposed to the off-transit spectrum (for both upper and lower quadrants separately and for the average spectrum), the solar spectrum convolved at the same resolution, and the spectrum of telluric water. This later is derived from a radiative transfer calculation in the atmosphere above the Paranal Observatory. The offtransit spectra have been convolved and shifted in order to fit the broadened stellar lines in the on-transit spectra, to ease the comparison and optimize the transmission spectrum calculation. Finally, the difference spectra between these on- and offtransit spectra are plotted at the top of Fig. 7. A modulation of the difference spectra at the level of $0.5 \%$ is observed. Half of this residual noise arises from fringing as said above. The other half shows the limit in manipulating the off-transit spectra to match the on-transit star spectrum. On the same figure (bottom), a fake line of $0.5 \%$ depth and $3 \AA$ width has been inserted. It is detected at the $3 \sigma$ level and illustrates our detectability limit. This upper limit is not in conflict with the HeI feature expected from the models $(0.25 \%)$, which however is a lower limit, not considering the full extent of the HeI exosphere. However, the relatively low content of helium in the exosphere of HD 209458b has to be interpreted against the large content of hydrogen measured recently (Vidal-Madjar et al. 2003). With this new evidence of an evaporated atmosphere around the hot planet, as was predicted previously (Schneider et al. 1998), the low abundance of helium must be explained.

\section{Conclusion}

This work aimed at estimating the detectability level of the HeI absorption line at $10830 \AA$ in the transmission spectrum of HD 209458b during an eclipse of the star by the planet obtained at VLT/ISAAC. To summarize the conclusions of this analysis:

- Data quality: the number of detected photons per individual spectrum is large, resulting in signal-to-noise ratios ranging from 380 to 690 on $2 \times 14$ spectra. One transit of HD 209458 was fully observed, in good external conditions. The comparison off-transit observations are of slightly worse quality. Unfortunately, we did not get spectra of comparison stars either during or after the transit,
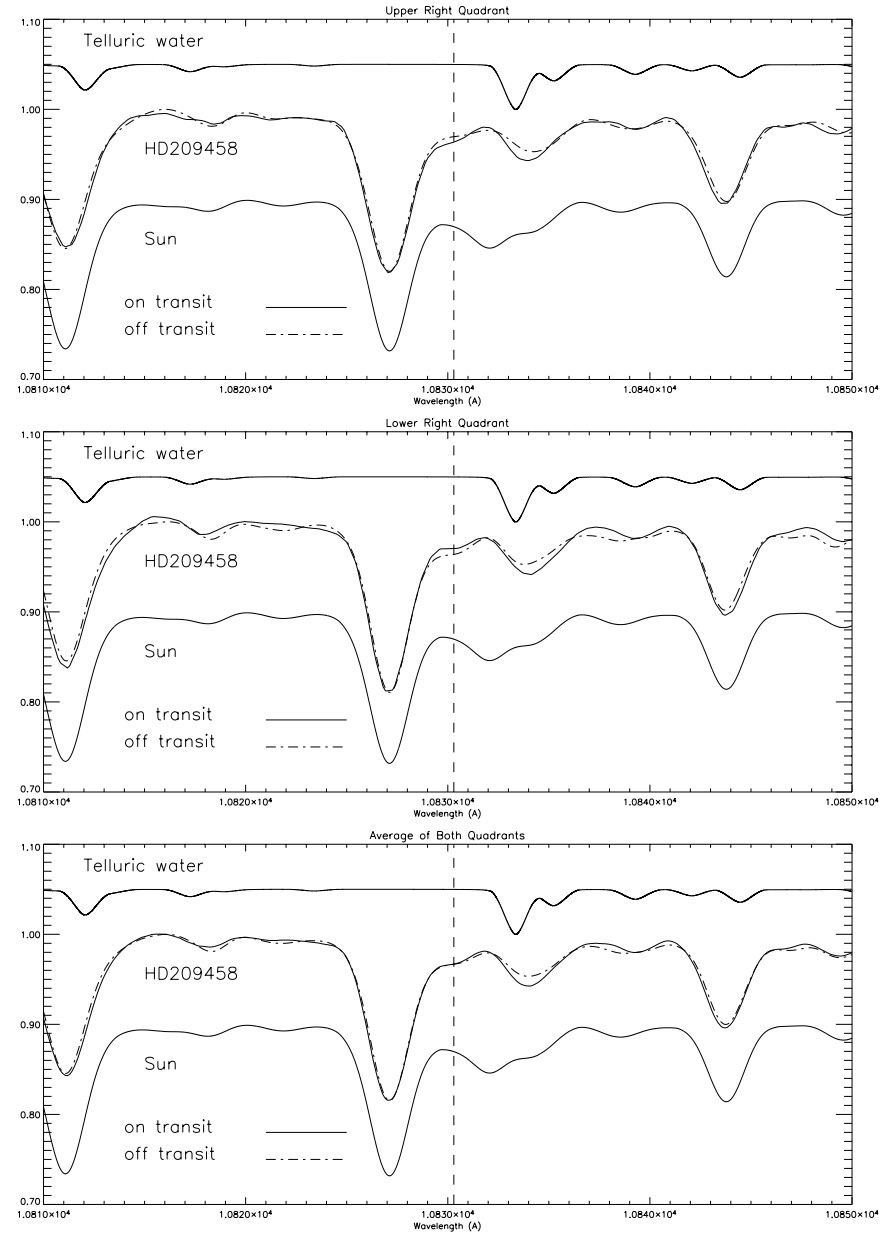

Fig. 6. After summation, in the planet velocity frame, of all on-transit spectra obtained on the upper and lower right detector quadrant, we show the comparison with reference spectra obtained off-transit (dotdashed line). Off-transit spectra were scaled to the on-transit spectraincluding a spectral convolution to get the same broadening of stellar line. The solar spectrum is also plotted for reference while the upper spectrum shows water absorption from the Earth atmosphere above the telescope; this is the result of radiative transfer calculation, convolved at the ISAAC resolution. The vertical line shows the position of the expected HeI feature. The spectra are all normalized to unity and shifted for a better visibility. An average of the upper and lower quadrant is also plotted. The bottom plot is the difference between on and off spectra, the thick line showing the average between upper and lower quadrants. The residual bumping in these difference spectra - at a level of $0.2 \%$ - is due to the limited precision of spectral convolution.

which prevents us from calibrating the variation of telluric features on other sources.

- Actual limitations of our search were mainly instrumental. The data suffered an odd-even column effect whose amplitude (a few percent) tends to vary with time, and with the position of the spectrum on the detector. Removing the pixel-to-pixel fringes in such a medium resolution spectrum proved efficient with a digital filtering, with a residual noise $0.2 \%$. Filtering also produces a loss in spectral resolution. Secondly, the proximity of the expected HeI feature to a telluric water feature ( $3 \AA$ apart), variable in time, also decreases the capability of producing a reliable reference spectrum of the off-transit observations. At such a 

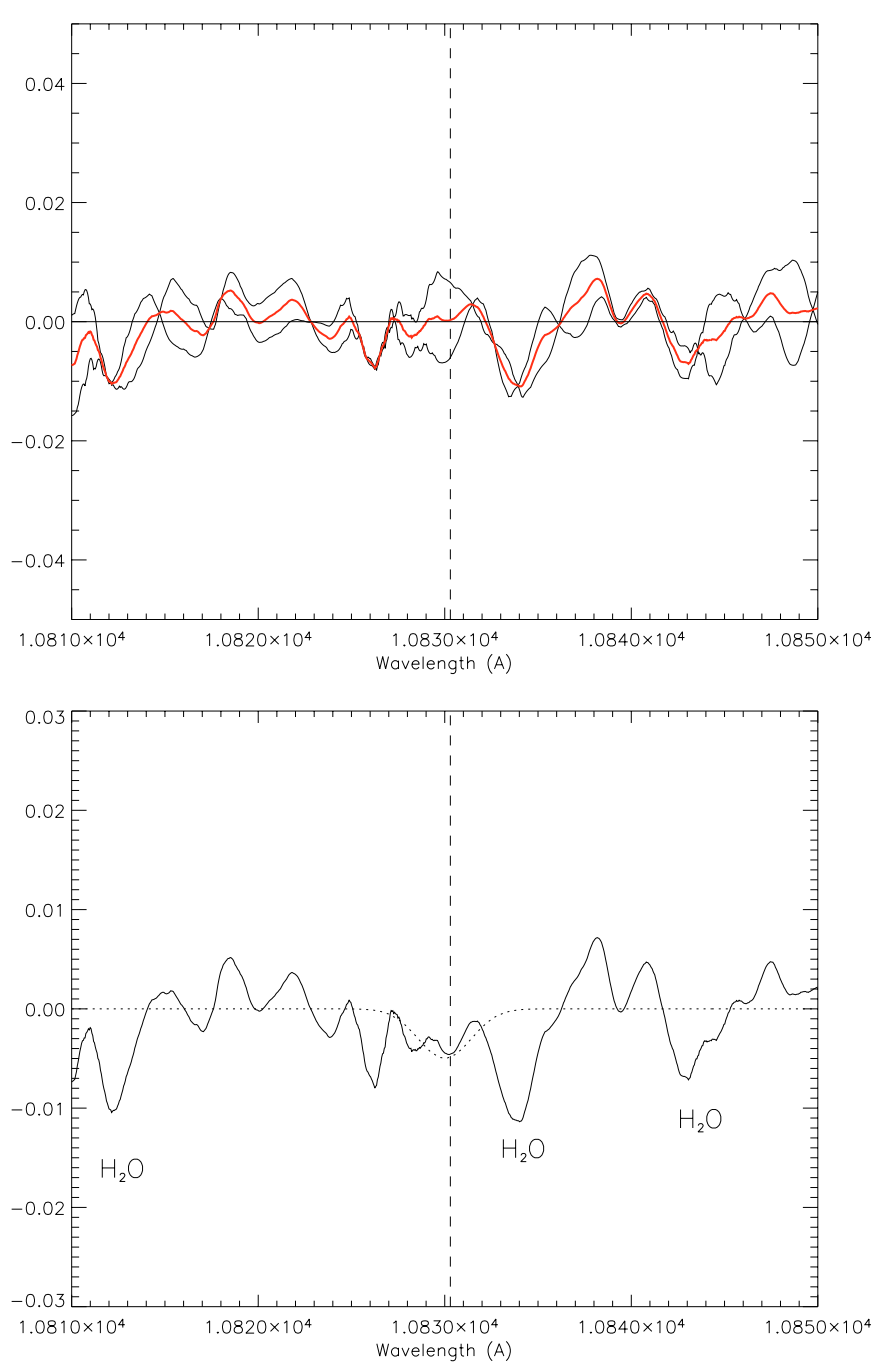

Fig. 7. The difference between on-transit spectra and off-transit spectra is shown (top). On-transit spectra were summed up in the planet velocity frame. The thick line in the top figure is the average of both quadrants, shown as thin lines. In the bottom plot, a fake absorption feature ( $3 \AA$ width, $0.5 \%$ central depth, dotted line) is inserted, illustrating the $3 \sigma$ detection limit. The difference spectrum is the average of both quadrants, the fake line being added to the on-transit spectrum.

spectral resolution and with the frequent blending of lines in the spectrum of HD 209458 as seen from the ground, it is difficult to eliminate the water features. The local noise is significatively enhanced.

- After centring the on-transit spectra in the velocity frame of the planet, the on-off difference spectra were calculated. A $3 \sigma$ upper limit of $0.5 \%$ deep absorption feature is then deduced at $10830 \AA$. This limit is compatible with the non-detection of the HeI absorption of the planet atmosphere $(0.25 \%)$. While it is probable that the exosphere contributes to the total absorption, this has not been observed within the detection capability range. We conclude that the atmosphere+exosphere absorption in the HeI feature is less than $0.5 \%$, which seems to be quite low with respect to what might be expected in view of the recent result on a large hydrogen content reported (Vidal-Madjar et al. 2003). This remains to be explained by models, because it would be atypical of a Jupiter-like planet, where the $\mathrm{He} / \mathrm{H}_{2}$ ratio is about 0.135 .

Future work in this direction could include:

- Acquiring higher spectral resolution data in the $1 \mu \mathrm{m}$ range, to avoid the line blending, or space observations in the near-IR domain to eliminate the telluric water problem. Avoiding the pollution of spectra with fringing at the data acquisition level is mandatory, as well as precisely monitoring the telluric variations during the transit sequence with the alternate observation of HD 209458 and a comparison star.

- Finding an instrumental configuration where fringes are neither so strong nor variable with space and time. The HeI feature is one of the easiest features to detect on the transmission spectrum of transiting atmospheres and exospheres, from the theoretical point of view, but this detection is sofar limited by instrumentation. In good instrumental and atmospheric conditions, it is foreseen that a feature as shallow as $0.1 \%$ should be detectable in an ISAAC spectrum of HD 209458 during transit.

- At a later time, developing a complete model for extra-solar exospheres and search for signatures of diffuse ionized gas surrounding the planet at larger height scales. Even if atomic and molecular compounds of the exosphere are less abundant than HeI, it could be that the larger cross section of the exosphere or/and a favourable location of the feature(s) over the star spectrum will allow an easier detection. For this, the atmospheric models of close-in extra-solar giant planets should include chemical and physical processes induced by the stellar wind, as suggested previously by several authors (Chassefière 1996, 1997; Schneider et al. 1998; Coustenis et al. 1997, 1998) and probably the atmospheric circulation (Showman \& Guillot 2002). Then new predictions could be tested for with an optimized instrumental setup.

Note that similar searches, in the optical range with echelle spectroscopy and other infrared spectrographs are on-going (Brown et al. 2002; Moutou et al. 2003; Richardson et al. 2003), which should produce in the mid-term precise constraints for atmospheric models of extrasolar giant planets.

Acknowledgements. We thank the Paranal Observatory service observers, the User Support Group and Quality Control at ESO for their efficient help. We are grateful to Thierry Fouchet for his useful advice in calculating the radiative transfer spectrum of telluric water vapour in the atmosphere above the telescope, to Thierry Lanz for discussing HeI physics and to Bruno Bézard for fruitful discussions.

\section{References}

Andretta, V., \& Jones, H. P. 1997, ApJ, 489, 375

Bundy, K., \& Marcy, G. 2000, PASP, 112, 1421

Brown, T. M., Charbonneau, D., Gilliland, R. L., Noyes, R. W., \& Burrows, A. 2001a, ApJ, 552, 699

Brown, T. M. 2001b, ApJ, 553, 1006

Brown, T. M., Libbrecht, K., \& Charbonneau, D. 2002, PASP, 114, 826 
Charbonneau, D., Brown, T., Mayor, M., \& Mazeh, T. 1999, November 22, IAU Circ., 7315

Charbonneau, D., Brown, T., Latham, D., \& Mayor, M. 2000a, ApJ, 529, L45

Charbonneau, D., Brown, T., Noyes, R., \& Gilliland, R. 2002, ApJ, 568,377

Chassefière, E. 1996, J. Geophys. Res., 101, 26039

Chassefière, E. 1997, Icarus, 229, 232

Cody, A.-M., \& Sasselov, D. 2002, ApJ, 568, 441

Coustenis, A., Schneider, J., Bockelée-Morvan, D., et al. 1997, in Planets beyond the Solar System and the Next Generation of Space Missions, ed. D. Soderblom, ASP Conf. Ser., 119, 101

Coustenis, A., Schneider, J., Wittemberg, R., et al. 1998, in Brown dwarfs and Extrasolar planets, ed. R. Rebolo, E. L. Martín, \& M. R. Zapatero Osorio, ASP Conf. Ser., 134, 296

Delbouille, L., Rolan, G., \& Neven, L. 1973, Photometric Atlas of the Solar Spectrum 3000-10000 ̊̊

Devillard, N. 2001, in Astronomical Data Analysis Software and Systems X, ed. F. R. Harnden, F. A. Primini, \& H. E. Payne, ASP Conf. Proc., 238, 525

Konacki, M., Torres, G., Jha, S., \& Sasselov, D. 2003, Nature, 421, 507

Marcy, G., \& Butler, P. 2000, PASP, 112, 137

Mayor, M., \& Queloz, D. 1995, Nature, 378, 355
Mazeh, T., Naef, D., Torres, G., et al. 2000, ApJ, 532, L55

Moutou, C., Coustenis, A., Schneider, J., et al. 2001, A\&A, 371, 260

Moutou, C., Coustenis, A., Schneider, J., et al. 2003, in Scientific Frontiers in Research on Extrasolar Planets, ed. D. Deming, \& S. Seager

Rauer, H., Bockelée-Morvan, D., Coustenis, A., et al. 2000, A\&A, 355,573

Rauer, H., Cameron, A., Barnes, J., \& Harris, A. 2000, DPS, 32, 32.09

Richardson, L., Deming, D., Wiedeman, G., et al. 2003, ApJ, in press

Schneider, J., Rauer, H., Lasota, J.-P., et al. 1998, in Brown Dwarfs and Extrasolar Planets, Proc. of a Workshop held in Puerto de la Cruz, Tenerife, Spain, 17-21 March 1997, ed. R. Rebolo, E. L. Martin, \& M. R. Zapatero Osorio, ASP Conf. Ser., 134, 241

Seager, S., \& Sasselov, D. 2000, ApJ, 537, 916

Showman, A., \& Guillot, T. 2002, A\&A, 385, 166

Tresse, L., Maddox, S., Loveday, J., \& Singleton, C. 1999, MNRAS, 310,262

Udry, S., Mayor, M., \& Queloz, D. 2000, in Planetary Systems in the Universe: Observation, Formation and Evolution, IAU Symp. 202, ed. A. Penny, P. Artymowicz, A.-M. Lagrange, \& S. Russel, ASP Conf. Ser.

Udry, S., Mayor, M., Naef, D., et al. 2002, A\&A, 390, 267

Vidal-Madjar, A., Lecalier des Etangs, A., Désert, J.-M., et al. 2003, Nature, 422,143 